\title{
ON THE FOCALITY OF MICROSCOPIC BRAIN LESIONS FOUND IN DEMENTIA PRAECOX
}

\author{
E. E. SOUTHARD, M.D.
}

Pathologist, Massachusetts Commission on Mental Diseases; Director, Psychopathic Hospital, and Bullard Professor of Neuropathology,

Harvard Medical School

BOSTON

THE IDEA OF MICROLOCALIZATION

A number of investigators of the mind and its diseases are now pursuing the study of the nervous system along lines roughly summed up in the term microlocalization. But this task must not be conceived narrowly as a task of the histoneurologist or the histoneuropathologist. Microlocalization in the nervous system and especially in the cerebral cortex has threads running to the broadest issues of physiology, embryology and psychology. Yet no one feels that psychologists, embryologists, physiologists, anatomists or even pathologists, in the narrower sense of the term, will solve the problems of microlocalization or even demarcate its problems for solution. When the idea of Flourens that the brain parts were mutually exchangeable in function like so many liver parts, was replaced with the ideas of topographic differentiation which we attach to the work of Hughlings Jackson and of Hitzig, it was as if the naked eye of observation was replaced with a lens of considerable power. The situation now is that this lens of great power, provided by the work of the embryologists, the physiologists and the clinical anatomists, must be replaced with a lens of still greater power, one that will permit us logically to face problems of microlocalization attaching to physiologic complications within the single gyrus. It has become a commonplace that the brain is a congeries of organs. It is not so well understood that each gyrus is a wonderland of structures and functions in which rich results are to be expected from hard but fascinating research.

As we look over the history of these developments, we of course find that Bevan Lewis had, in advance of his time, laid down the idea of cell differentiation within the cerebral cortex, clearly distinguishing the large cells of the motor cortex, for example. In much of the work of Meynert and Flechsig, the idea of microlocalization can be discerned. However, even in the embryological considerations of Flech- 
sig, the notion of the gyrus as little more than a functional unit is the prevailing idea. Decades were devoted to the isolation of one neuron after another by ingenious technical methods and to developing the conception of the nervous system as a mass of embryologically and functionally separate units, expressed, for example, in the neuron hypothesis of 1891 . Listeners to the lectures of Nissl became familiar with his ideas of the structural and functional differentiation of the various cortical parts.

The idea of microlocalization scarcely flourished to the full in the last decade of the nineteenth century, however, since workers were engaged in assimilating the principles of Weigert concerning neuroglia and in trying to find qualitatively the same sort of lesions in the nervous system that they were finding in other organs. In fact, this search neatly culminated in the establishment in the years 1899-1904 of general paresis as a definite example of chronic inflammation of the nervous system. The pathologist was here trying to find, and succeeded in finding, certain lesions scattered in different parts of the nervous system, and lesions of qualitatively the same sort throughout. Nor, in the rush of new ideas, was the material in general paresis used to the utmost in throwing such light as it might on the problem of microlocalization. Thus, had the world been seriously trying to find out the nature and genesis of mental phenomena and symptoms, it would surely have executed elaborate studies in the distribution of the characteristic paretic exudate, with the idea of correlating these local variations in exudate with processes and symptoms. The issue of such an investigation could have been only positive or negative. As it stands, the situation is doubtful. In fact, it may be confidently doubted whether the paretic exudate itself is at all responsible for many of the characteristic symptoms in paresis, for example, grandiosity, amnesia or even dementia, symptoms which are not found to correspond even roughly with the amounts of exudate discovered in the brains. ${ }^{1}$ So far as paresis is concerned, very possibly it was more important to determine it to be syphilitic and to embrace eagerly the opportunities afforded of a Wassermann reaction than to pursue seemingly recondite studies in microlocalization. Meantime the topographic anatomists were laying down the results which the pathologist could at last employ without signal loss of time.

When the interesting and fundamental work of Hammarberg appeared on the histology of freeblemindedness in 1895, it was plain

1. Southard, E. E.: A Comparison of the Mental Symptoms Found in Cases of General Paresis With and Without Coarse Brain Atrophy, J. Nerv. \& Ment. Dis. 43:204, 1916. 
that this Swedish investigator had had to spend more than half his time, not on the histopathology of feeblemindedness, but on normal histology. The situation is now greatly improved with the work of Elliot Smith, who showed that even the naked eye will, under proper conditions, detect striking differences between the gyri of different parts of the brain on section, and especially with the work of Campbell, who, after an extensive study of the histology of the different gyri, has offered a valuable atlas with a store of functional comments and speculations.

Even before Campbell, Bolton had laid down exact lines concerning the histology of different parts of the occipital region, and had contributed the idea of visuo-sensory and visuo-psychic regions therein. Similar work has been done by Brodmann, in Germany, who however feels that functional speculations are hardly in point at the present time. Visitors in the private laboratory of Vogt in Berlin some years since must have been impressed with the tremendous elaboration of structures which could be demonstrated in the different parts of the brain, even by so simple a technical method as the method of Weigert for myelin sheaths. For decades, also, the world has been watching the development of the ideas of Ramon y Cajal, whose labors stand not second to those of Bolton, Campbell, Brodmann, and the rest of the topographers.

In fact, the research situation is theoretically so promising in view of the progress along all these lines by workers in different countries, that it is a little surprising that so little neuropathologic application has been made of the results as attained. There seems to be a curious lack of tempo in neuropathologic research. Bevan Lewis' ideas of cortex differentiation came, it would seem, too early to affect the main current of investigation. The work of Donaldson in 18891890 on the brain of Laura Bridgman has not been followed up by any considerable series of similar investigations, and the Laura Bridgman work apparently led its author to deeper considerations of growth and development rather than to progress along the line of neuropathology.

\section{ANTILOCALIZING VIEWS OF PSYCHOLOGISTS}

Again, late in the nineteenth century, there prevailed the Wundtian psychology. The reader of Wundt's analysis of aphasia, for example, is convinced that, despite the profound distinctions made, the philosopher was no longer a physiologist and set himself the task of anti-localization in the spirit of a system-maker rather than that of an independent seeker after facts and independent interpretations. The idea of the unity of the mind, a very fine-grained and closely interwoven unity, has prevailed in many quarters, perhaps in all quarters, dominating research. To these psychic unitarians it would 
have seemed almost a pity if any one could show, for example, an identity of speech processes with processes of the left inferior frontal gyrus. To the conservative wing of the psychic unitarians was added the force of those strictly scientific persons who feel that a fact is a fact and a speculation is necessarily a bad speculation. Thus, for example, if the simpler ideas of Broca concerning speech localization ought to be modified by the modern concepts of Marie, then this is scored as a victory by those holding to the adamantine unity of the mind, on the one hand, and by those who are sceptical of all scientific results in the psychic field of whatever sort.

Added to this situation was the Freudian tendency. Disregarding the truth or falsity of the Freudian contentions, it is clear that his followers are engaged much more in finding satisfactory categories of a logical nature in which to place globar tendencies shown by their patients than they are in conceiving the brain functional lines along which an hysterical dissociation might proceed, a repression be mechanized, or a censor be enthroned.

Inasmuch as Descartes made a laughable error in assigning the seat of the soul to the pineal body, it is best for the modern to "play safe" by failing to notice both the pineal body and the brain itself and to solve the problem either by denying that it exists or by failing to consider it at all. One of these modern worthies assured me some years ago that there was no doubt a correlation between mental processes and brain processes and that, theoretically, the "needle" of demonstrable lesions could be found in the "haystack" of neuronic brain systems, but that for his part he regarded that problem as theoretically solved and desired to get on with something genuine and practical.

Accordingly, the academic psychologists, if we may so term the Wundtians, and the apparently more radical Freudians, have alike little interest in the matter of microlocalization, that is, in the correlation of mental with brain processes, either normal or pathologic

It is, therefore, almost with a feeling of being alone in the wilderness that one endeavors to work in the direction of neuropathologic correlation; either there is no problem or it is theoretically settled beforehand. A psychiatrist of light and leading asked me one day what, after all, I was about in working along structural neuropathologic lines. Anybody with a logical turn of mind and any capacity for observation ought rather to be busily applying the modern categories of psychopathology to clinical psychiatric and neurologic material. If such doubt of the fundamental value of this kind of work could prevail in the mind of a leading psychiatrist, I felt that I could do no better than bring these general ideas before the Association of American Physicians. 
It is, as possibly throwing light on the problems of microlocalization, that the material in dementia praecox struck me as of value. To be sure, the world was full of a rather empty discusison as to whether dementia praecox was an organic or structural disease. Any one who professes to find brain changes in dementia praecox was perforce regarded as a controversialist, engaged in demolishing the pet ideas of some workers who wanted to show that dementia praecox was a disease of maladaptation or a disease with so-called Freudian mechanisms. Let me say, therefore, that to embark on studies of the brain in dementia praecox and to find brain changes therein is not at all to deny that dementia praecox is an instance of maladaptation of the individual to his environment and not at all to deny the possible importance of Freudian mechanisms in the disease. ${ }^{2}$

\section{ANOMALIES IN DEMENTIA PRAECOX BRAINS}

In $1906, \mathrm{I}$ began to study the brain problems of dementia praecox and collected first-fruits of that study at the Medical Congresses in $1910^{3}$ and 1913.4 I then showed that the brains of dementia praecox subjects were extremely likely to show evidences of anomaly, that even when there was no anomaly, there were very likely to be microscopic changes. To be specific, I found twenty-three out of twentyfive carefully studied brains to be significantly anomalous. I do not mean that the anomalies shown were visible the whole length of the demonstration hall, as they are apt to be in brains of the feebleminded; but I mean that the asymmetries and irregularities were of a demonstrable and photographable nature. Friendly critics offered the suggestion that similar anomalies were to be found in brains of normal persons. Unluckily, the brains of normal persons, in large numbers, are not available so far as I know in any laboratory in the world, at least in such a state as to permit proper photographic analysis. However, I think I am able to answer these critics by showing that the brains of subjects of the so-called manic-depressive psychosis show such anomalies in very few instances. ${ }^{5}$ Using certain criteria, I found four fifths of my dementia praecox brains to show such anomalies and but one fifth of the manic-depressive brains similarly affected.

2. Southard, E. E.: The Mind Twist and Brain Spot Hypotheses in Psychopathology and Neuropathology, Psychol. Bull. 11:117, 1914.

3. Southard, E. E.: A Study of the Dementia Praecox Group in the Light of Certain Cases Showing Anomalies or Scleroses in Particular Brain-regions, Proc. Am. Medico-Psychol. Assn., May, 1910.

4. Southard, E. E.: On the Topographical Distribution of Cortex Lesions and Anomalies in Dementia Praecox, with Some Account of their Functional Significance, Am. J. Insan., October, 1914, and January, 1915.

5. Southard, E. E.: Anatomical Findings in the Brains of Manic-Depressive Subjects, Boston Med. and Surg. Jour., 1915, 172: No. 4. 
But it is one thing for a brain to be anomalous and another for it to be functionally disordered. The underlying hypothesis here is that the anomalous regions of the brain are in some sense weak places therein, such that disease of a toxic or metabolic nature, for example, at puberty, may unfavorably affect the anomalous and poorly constructed region. These considerations I presented with some histologic confirmation at the 1914 meeting of the Association in a paper "On the Direction of Research As to the Analysis of Cortical Stigmata and Focal Lesions in Certain Psychoses." Further reflection on this finding led me to the considerations about tissue decomplication developed at the 1915 meeting. ${ }^{7}$

But it was clear that the few cases of normal-looking brains in dementia praecox formed the crux of the situation, since the critics of material from anomalous brains might properly charge the investigator with inability to cull out the acquired from the inborn lesions. I therefore determined to study with sufficient intensiveness the brains of cases of dementia praecox that showed no anomalies or scleroses. In connection with this study, five brains were investigated, four of them looking in the general direction of dementia praecox and one of them in that of manic-depressive psychosis. These five brains were the residuum of a series of 120 coming from psychoses of all sorts.

Before I could bring the topographic problem into clear relief, I found that in the analysis of these five brains, the problem of parenchymatous versus interstitial lesions stood out. At the 1916 meeting I presented the conception that in the nervous system there should be a dissociation of lesions somewhat similar to that in the kidney. ${ }^{8}$ To be sure, the parenchymatous and interstitial lesions of the kidney are, as is well known, often if not almost always commingled. But the fact that the lesions in these two types of renal tissue are so commingled in a given case militates not at all against the idea that there are, roughly speaking, two kinds of nephritis - parenchymatous and interstitial. The same theoretical situation holds in the nervous system. I found that parenchymatous and interstitial lesions could be dissociated and combined in the nervous' system, much as similar lesions could be dissociated and combined in the kidney. To be sure,

6. Southard, E. E.: On the Direction of Research as to the Analysis of Cortical Stigmata and Focal Lesions in Certain Psychoses, Tr. Assn. Am. Phys. 29: 1914.

7. Southard, E. E.: Advantages of a Pathological Classification of Nerve Cells, with Remarks on Tissue Decomplication as Shown in the Central and Cerebellar Cortex, Tr. Assn. Am. Phys. 30: 1915.

8. Southard, E. E.: On the Dissociation of Parenchymatous (Neuronic) and Interstitial (Neuroglia) Changes in the Brains of Certain Psychopathic Subjects, Especially in Dementia Praecox, Tr. Assn. Am. Phys. 31: 1916. 
the parenchyma of the nervous system was a parenchyma composed of neurons and their adnexa and the interstitial tissue of the nervous system was complicated by the fact that a great deal of it is neuroglia tissue, embryologically different from the interstitial tissue of the kidney. Nevertheless, it is well known that many of the properties of neuroglia of ectodermic origin are similar to the properties of mesodermic interstitial tissue. The alterations of the mesodermic interstitial tissue also existent in the nervous system are of far less consequence than those of the neuroglia tissue. The one case of manicdepressive psychosis, intensively examined, failed to show convincing degrees of parenchymatous lesion, but the dementia praecox case had well-marked parenchymatous disorder, that is, varying degrees of cell loss, to which the interstitial reaction, that is, gliosis, was found to be not at all proportionate. The result of this study seemed to be that parenchymatous disorder, namely, cell losses and interstitial disorder, namely, gliosis, must be investigated separately if we are to make headway in this group of cases. Thus it would be decidedly unwise to argue from the data of neuroglia preparations that the gliosis of such and such an area was an indicator of parenchymatous loss. To be sure, the gliosis is probably an indicator of something of general or local significance in the brain. But no facile application of the principle suggested by Weigert's neuroglia work could safely be made. Even if gliosis, as a rule, signifies some degree of wear and tear on the part of the parenchyma of the part affected, yet there was no good evidence that the gliosis was at all proportionate to the parenchymatous wear and tear.

STRATIGRAPHICAL ANALYSIS OF THE CEREBRAL CORTEX

Having thus shown that the brain conditions roughly corresponded to, for example, renal conditions, and that the histopathologic analysis of injured brain tissues must look in both these directions in search of successful correlations, I proceeded to a stratigraphical analysis of the finer cortex changes in these brains, presenting results at a meeting of the American Neurological Association. ${ }^{9}$ I tried to study separately the nerve cell losses, on the one hand, and the gliosis (including satellitosis), on the other hand, and then to learn whether the character of these changes in the upper and lower layers of the cortex was of any functional significance. For convenience, I wish to term the upper layers of the cortex supracortical and the lower layers infracortical. In fact, it might be well at times to speak of the supra-

9. Southard, E. E.: The Stratigraphical Analysis of Finer Cortex Changes in Certain Normal-Looking Brains in Dementia Praecox, J. Nerv, \& Ment. Dis. 45:97, 1917. 
cortex and the infracortex. The comparative anatomists seem to have demonstrated the importance of some such distinction and the literature has for some time contained references to the so-called suprastellate (supragranular) and infrastellate (infragranular) layers of the cortex. Omitting controversial details, it appears that almost all workers are committed to the idea that the majority of the supracortical structures are of more recent evolution than the majority of the infracortical structures. As many of the mental functions in man are of recent evolutionary origin and as all signs point to the cerebral cortex as somehow engaged in these processes, it seems natural to assume that the supracortical layers, or what we may term the supracortex, are the basis of many of these higher psychic functions. With the same plausibility, we may argue that the infracortical layers, or what we may term the infracortex, is the basis of such mental or quasi-mental functions as are found in those animals possessing an infracortex and not yet a supracortex.

All these interpretations are independent of any topographic conceptions, and for the purposes of generalization one neglects for the moment the important distinction of the archipallium and neopallium, developed by Elliot Smith.

Taking the whole cortex, by and large, modern work seems to me to invest the supracortex with higher psychic functions than the infracortex.

Now in a disease like dementia praecox as psychologically formulated by Bleuler, we find the main feature to lodge in what Bleuler terms schizophrenia. This most valuable term expresses what one might call "split-mindedness" or mental dissociation. Much of modern psychology, whatever may be said to the contrary, is founded on the work of the associationist school of, for example, John Stuart Mill. We should hardly get on without some of Mill's so-called mental chemistry. Well, for better or worse, associative processes must have their obverse in dissociative processes, and the measure of mental dissociation is the degree of schizophrenia in Bleuler's sense. Kraepelin himself, who, on purely clinical grounds of a combination of certain symptoms in certain ways, laid down the conception of dementia praecox, has so far admitted the validity of Bleuler's conception of schizophrenia as to use the term schizophrenic very frequently in his description of patients. For one of the most exquisite examples of schizophrenia, namely, the finely divided cleavage of speech known as Wortverwirrtheit, Kraepelin has constructed the term schizophasia on the analogue of schizophrenia.

We mean then by schizophrenia a process of mental dissociation, a psycholytic or ideolytic process. The cleavage may lie between the intellect and the emotions, such that the emotions do not at all fit the 
ideas entertained by the patient. Or the cleavage may lie between the emotions and the will, and a bizarre-looking conduct may fit a certain quasi-normal state of feeling. But the cleavage is not necessarily so elective and total. The cleavage may be within some particular train of thought. Here is schizophrenia in its best display. Instead of the regular train of thought, we may have a train of thought like a telegraph despatch, or even a train of thought suggesting in its obviously inappropriate sequence a cipher or a set of types reduced to pi. But absolutely pied thinking is not the rule and the so-called word salads are often but passing phases in the development of a dementia praecox course.

\section{MANY EFFECTS OF MENTAL DISSOCIATION PROBABLY CORRELATED WITH SUPRACORTICAL DISEASE}

Here is not the place to expound or to describe schizophrenia. Inasmuch, however, as many of the schizophrenic effects that strike the observer as so bizarre in dementia praecox thinking are probably correlated with the operations of the outer layers, that is, of the supracortex itself, we have to inquire whether cases of this order show lesions preferably in the supracortex. ( Of my four cases of dementia praecox, all showed lesions in the supracortex in the nature of cell loss and of more or less disproportionate gliosis, except one case.) This case was one approximating the so-called paranoia, a disease perhaps allied to dementia praecox, but often running years or throughout life without any of the bizarre psycholytic, schizophrenic, thought-splitting phenomena here under discussion. For paranoiacs of this rare description (there may be in a dozen institutions in Massachusetts at this time not more than twenty-five or thirty of these particular cases), present quasi-normal appearances that have been described sometimes as psychic malformations and often give the impression of ability to fit in to a reconstructed world with the patients in the center as Kaisers or I. W. W. chiefs therein. In short, many of these paranoiacs, victims of the severest and most intractable psychosis known, are nevertheless without a trace of mental splitting such as I have hinted at above. And, in fact, the paranoiac in question showed no signs of mental dissociation, but rather showed signs of what might be termed a hypersynthesis of such facts in her environment as fitted her morbid ego and her jealousies. What I am getting at is that the psychopathologic analysis of this case of paranoia precisely did not demand any evidence of supracortical disease, in fact, had there been supracortical disease of any moment, we should have wondered whether there had not been some disintegration of the patient's personality, at any rate at the close of her life. It is interesting that this particular paranoiac did show some infrastellate dis- 
ease which, as will be mentioned below, I regard as correlated with the auditory hallucinations which she showed.

The other three cases intensively examined ( $\mathrm{I}$ may recall that notes were made as to different types of cell loss, gliosis, satellitosis, vascular changes, etc., for each layer in each of from twenty-five to thirty areas of the cortex in each subject) showed signs of supracortical disorder as well as of infracortical disorder of varying degrees in different parts of the cortex, and all three of these cases showed signs of schizophrenia. This evidence is at least suggestive that supracortical disorder is necessary in schizophrenia. I felt satisfied in my own mind, from the results of the work, that not only is the cerebral cortex the proper study of the psychologist and the psychiatrist, but that the supracortex is in a still more intimate sense the proper study of the psychologist or psychiatrist who has to deal with the higher functions of what may be called psychic association or synthesis. To my mind, such studies, if in future confirmed, will throw an important added light on the problems, already partly illumined by comparative anatomy.

Let me insist here, if it is not superfluous, on the fact that I am not proposing an hypothesis to the effect that every time a mental split occurs, a cell drops out of the supracortex. I do not even see in my mind's eye a dendrite dropping off a supracortical cell to signalize the cleavage of an idea. There is very properly much fun to be made of workers who regard ideas as fine transparent substances poured in and out of nerve cells as wine in and out of goblets. Aside from the problem of parallelism and interactionism suggested by the figure that regards brain cells as vehicles for ideas, my own contentions deal rather with the observational facts. I would regard all gliosis as merely indicating and not defining the type of cortical disease in question and I should regard the nerve cell losses or other changes as likewise nothing but indicators of the kind of thing going forward, or perhaps as indicators that something or other is going forward in the injured tissue. To be sure, I would think that destructive brain changes would be more likely to be associated with mental dissociation (with schizophrenia, gliosis) than with other types of synthetic or redistributive mental processes. Supracortical neuron destruction, to put the whole matter in a formula, is to my mind correlated with schizophrenic processes. What, you may well ask, is to be correlated with hypersynthetic, falsely synthetic or redistributive mental processes of the mind twist sort? For the present I, for one, have no idea what cortical process corresponds to these nondestructive, morbid psychic processes. Mind lack and mind loss should correspond with cell lack and cell loss. What we term figuratively mind twist, for example, false reasoning without loss of sensory intake and power, without 
loss of memory images, without loss of power of expression, without any evidence of coarse loss of function, remains as a process not readily statable. Perhaps we must fall back here on the idea of physicochemical changes and of anomalous distributions of energy in normal neuronic systems. But these are speculations so remote from observational facts that so far as I. can see they cannot even do the observer any harm.

\section{CORRELATIONS OF HALLUCINOSIS AND CATATONIA WITH INFRACORTICAL DISEASE}

Besides schizophrenia there are other bizarre effects in dementia praecox. Perhaps the strangest thing in the world is catalepsy. Catalepsy, catatonia, flexibilitas cerea, are not infrequent symptoms in many cases of dementia praecox. They all have the character of muscular hypertension somewhat suggestive of hypnotic effects or again of the effects of drugs on the muscular system. Sometimes the phenomenon of negativism and resistivism reminds one of the Sherringtonian experimental effects showing innervation of antagonistic muscle groups. Possibly these processes are to be explained on some general line as schizophrenia; possibly they are due to some kind of lysis; some workers are inclined to use the concept of inhibition very freely in the attempt to explain them. They are often a transient phase recurring again after comparatively normal intervals in a schizophrenic patient, and it seems impossible to evoke them by any form of stimulation of the patient.

In a general way, such conditions as catalepsy seem a good deal more like phenomena producible in lower animals than would schizophrenia. Some of the hypnotic or pseudohypnotic appearances in lower animals roughly resemble the catatonic effect. It was in a case of catatonia that Alzheimer first stated that there were nerve cell changes in dementia praecox, and it will be remembered that he placed these changes (chiefly gliosis) in the lower layers of the cortex. To a certain extent, my own investigations here confirm those of Alzheimer; at all events, in the cases showing catatonia there were lesions in the infracortex.

Another symptom not at all so bizarre as schizophrenia and catatonia is hallucinosis. Hallucinations are rather quasi-normal effects that the normal person rather readily understands and that occur in a great number of mental diseases. Hallucinosis is in short a far less pathognomonic symptom for dementia praecox than is either schizophrenia or catatonia. Many forms of hallucination seem to be of so simple a nature that they would well be elicited in the lower animals, could we only get at the psychic interiors of the lower animals to learn their mental contents. A priori, therefore, it seemed to me that 
the disease process underlying hallucinosis ought to be in the infracortex rather than in the supracortex, and there was some confirmation of this idea in the case studied. I mentioned in the foregoing the fact that late in the disease, the paranoiac patient developed auditory hallucinosis. She showed infracortical cell loss of a mild but distinct degree. It will be remembered that the brain of this case was the most nearly normal of all the brains so far studied in the whole series.

The stratigraphical analysis of these brains accordingly proved very alluring. I seemed to get evidence that the supracortex was a region whose disease might well spell schizophrenia, whereas the infracortex was a region whose disease was related with lower level symptoms, such as catatonia and hallucinosis. The supracortex and the infracortex would then be regions of differential interest for the psychiatric investigator, who would seek in the supracortical region for evidences of higher intellectual disorder in the field of association, combination and abstraction, and in the infracortex for evidences of lower forms of mental disorder, such as catalepsy and hallucinations. I would not stick on the question of higher and lower functions at this point. Catatonia may well be a far more complex matter than many forms of intellectual dissociation; I call it lower because it seems to me that a simpler organism could show catalepsy rather than disorders of combination and abstraction in the intellectual field.

I have recently reviewed the cell findings in the different cortical areas, assuming that I should find rather suggestive topical correlations between special kinds of symptom and special loci. I assumed that a priori no one would be likely to hunt for the source of auditory hallucinations in the smell zone as defined by the comparative anatomists, and that one would hardly be likely to look for the basis of visual hallucinations in the superior temporal gyrus!

Perhaps in accordance with the opinion of the learned psychopathologist above mentioned, it is a priori certain that in point of fact auditory hallucinations have their basis somewhere in the temporal lobe and visual hallucinations somewhere in the occipital region, and why undertake a superfluous task of setting out exactly where and under what conditions they occur? We ought to be helped greatly in this matter by the focality of lesions in dementia praecox.

TENDENCY TO LOBAR AND LOBULAR FOCALITY OF LESIONS

In the beginning I had been attracted by the fact that there was often a lobar or lobular hypoplasia, atrophy or sclerosis in these brains. In fact I had tried, reasoning from the gross data alone, to draw tentative conclusions as to the main lines of functional differentiation in the symptoms of dementia praecox. 
Noting how difficult was the distinction between inborn anomaly and acquired lesion and how uncertain one might be that a given hypoplasia had anything to do with a given disorder of function, I felt that I ought perhaps to investigate cases without gross lesions.

Now one of the most generally significant results of this, intensive examination of numerous areas and all layers in dementia praecox brains is that there is a certain focality in the microscopic lesions. I still find the focality to be rather a lobar or lobular one than an intragyral focality. To be sure, my colleague, Dr. H. I. Gosline, has made some interesting observations on some exquisitely focal lesions of intragyral distribution, possibly related with tuberculosis, in certain dementia praecox brains. ${ }^{10}$ Gosline has endeavored to show that the situation of these lesions (demonstrable by fat strains) is such as to fit with my own older contentions as to the relation of certain symptoms to particular regions. Notably Gosline endeavors to confirm my idea of the correlation of catatonia to postcentral lesions. My own work, as here reported, deals not with results of fat stains, but with the results of the study of cell losses by the ordinary tinctorial methods for determination of tubercle, at least with nerve cells (cresyl violet after formalin fixation). I have tried to show the cell losses thus made out as a little more reliable than the fat-stained cells and deposits that Alzheimer, Cotton and Gosline have used. Using the methods of these observers, I obtain too rich a display of lesions, as a rule, to permit correlation.

The kind of focality of lesions which I believe to have demonstrated in these brains is not intragyral, but lobar and lobular, or in certain cases a focality of lesions affecing the whole gyrus. By and large in these brains, as one examines the different layers within a gyrus, the. layers are apt to show throughout the gyrus the same kind of lesions, whether gliosis or cell losses. In short the focality of these lesions is not the focality of tubercles, tumor masses, glanders lesions or exudative lesions. It is rather a focality such that, for example, the postcentral gyrus on one side is affected in a certain way throughout the gyrus with such and such layers homogeneously affected. The postcentral gyrus of the other side is relatively normal.

What now are the functional results of a study of the topographic distribution of the lesions just mentioned? Can we by focalizing attention on certain gyri discover functional correlations of lesions with certain symptoms?

10. Gosline: Paresis or Dementia Praecox? Boston M. \& S. J. 177: No. 10, 1917. 
The functional correlations of my dementia praecox studies published in 1910 and 1914-1915 were summed up from the topographical point of view as follows:

1. Delusions are, as a rule, based on frontal lobe disease.

2. Catatonic symptoms are, as a rule, based on parietal lobe disease.

3. Auditory hallucinosis is, as a rule, based on temporal lobe disease.

Re the frontal lobe correlation with delusions, I found an exceptional group of delusional cases without frontal emphasis of lesions; but I found a plausible reason for these exceptions. On analysis, the nonfrontal cases of delusion formation turned out to be cases with a tendency to what may be termed hyperphantasia, that is, to an elaboration of phantasies not more than half believed, or at any rate not fully believed, by the patient. It is clear that from what we know of the probabilities of localization in the brain, both from a comparative and anatomoclinical point of view, the parietal lobes might plausibly be implicated in the function of imagination, and that parietal disease might well be attended with functional disorders of imagination, such as overimagination or what is here termed hyperphantasia.

It is plausible to argue that because the parietal tissue, newly evolved in the higher animals, lying between the tactile postcentral region and the visual occipital region and the auditory temporal region, should be a tissue related with apparatus for combining these sensory data, a tissue which might unobjectionably be termed a center for combining percepts and concepts involving two or more forms of sensation or perception. Whatever the merits of this a priori contention, at all events I found a tempting correlation of phantastic delusions with parietal lobe atrophy.

I summed up the anatomic situation as follows:

On the whole, the correlation between delusions and focal brain atrophy (or aplasia capped by atrophy?) is very strong, particularly if we distinguish (1) the more frequent form of delusions with frontal lobe correlations from (2) a less frequent form with parietal lobe correlations.

The nonfrontal group of delusion formations, the writer wishes to group provisionally under the term hyperphantasia, emphasizing the overimagination or perverted imagination of these cases, the frequent lack of any appropriaate conduct-disorder in the patients harboring such delusions, and the a priori likelihood that these cases should turn out to have posterior association center disease rather than disease of the anterior association center. This anatomic correlation is in fact the one observed.

My previous work had also suggested a possible correlation between catatonic phenomena and parietal (including postcentral) disease. Ten of fourteen definitely catatonic cases yielded gross lesions in the 
parietal or other post-rolandic regions. Of the remaining four, two, negative in the gross, proved to be microscopically altered in the parietal region, and there were indications of a correlation in the remaining two cases. It must be remembered with respect to correlation that I am using the term "catatonic" here as the name of a symptom. I am not offering a correlation between anatomic or microscopic lesions and the catatonic form of dementia praecox.

For example, the symptom flexibilitas cerea is not necessarily an indicator that the victim belongs in the so-called catatonic form of dementia praecox, despite the fact that flexibilitas cerea is one of the prettiest examples of catatonia ever described. Four of the five cases in the 1914-1915 series that showed flexibilitas cerea had parietal anomalies or atrophies, and the fifth case, though it was entirely negative in the gross, was one which showed an extreme degree of satellitosis in the postcentral region examined microscopically. These observations and certain a priori considerations led me to formulate the idea that catatonia, and particularly flexibilitas cerea, was a form of disorder of kinaesthesia.

$R e$ the correlation of auditory hallucinosis with temporal lobe lesions, it is easy to see that such a correlation ought to exist if any gross correlations at all were expected. In point of fact, nine of my twelve hallucinating cases have had temporal lobe atrophy or aplasia. One of the three remaining cases had ample microscopic changes in the temporal lobes; one was clinically somewhat doubtful and, in fine, only one of the twelve hallucinating cases could be safely said to have neither gross lesions nor important microscopic lesions in the temporal area.

To sum up then: (1) we expect frontal lobe lesions in cases of delusion formation, provided that the delusion formation is not of the phantastic and overimaginative sort, whereupon parietal lesions would be expected; (2) we expect parietal lesions associated with catatonic symptoms, and (3) we expect temporal lobe lesions in cases of auditory hallucinosis.

\section{ANALYSIS OF FOUR CASES}

Accordingly, I have collected the microscopic data in the four cases at present under discussion under these heads-frontal, parietal and temporal. For convenience I have divided the cortex into three portions - frontal, parieto-occipital and temporosphenoidal. The following table indicates the extent of nerve cell loss and of neuroglia proliferation, separately in the three regions mentioned:

It is clear from inspection of the table that Case 1 (10.9) stands out as a case in which the parenchymal disease is especially parietooccipital, since in ten loci seventeen instances of nerve cell losses in 
different layers were found. Here then should be a case with catatonia unless perchance the lesions in the parieto-occipital area should be exquisitely occipital and not affect kinesthetic areas. In point of fact the lesions enumerated are all in the postcentral, superior and inferior parietal areas and are especially marked in the inferior part of the postcentral gyrus and in the superior parietal region on both sides.

Showing Extent of Nerve Cell Loss and of Neuroglta Proliferation

\begin{tabular}{|c|c|c|c|c|}
\hline & $\begin{array}{c}\text { Case I } \\
\text { (10.9) } \\
\text { Duration } \\
14 \text { Yrs. }\end{array}$ & $\begin{array}{c}\text { Case II } \\
\text { (12.41) } \\
\text { Duration } \\
\text { 10 Yrs. }\end{array}$ & $\begin{array}{c}\text { Case III } \\
(12.47) \\
\text { Duration } \\
2 \text { Yrs. }\end{array}$ & $\begin{array}{c}\text { Case IV } \\
(11.36) \\
\text { Duration } \\
20 \text { Mos. }\end{array}$ \\
\hline Frontal loci...$\ldots \ldots \ldots \ldots \ldots$ & 9 & 9 & 9 & 6 \\
\hline Nerve cell losses ... & 5 & 44 & 35 & 34 \\
\hline Neuroglia proliferation & 7 & 8 & 4 & 3 \\
\hline Parieto-occipital loci ...... & 10 & 9 & 10 & 7 \\
\hline Nerve cell losses ...... & 17 & 47 & 54 & 19 \\
\hline Neuroglia proliferation .... & 8 & 3 & 2 & 8 \\
\hline Temperosphenoidal loci .. & 10 & 10 & 10 & 6 \\
\hline Nerve cell losses ...... & 9 & 48 & 50 & 25 \\
\hline Neuroglia proliferation...$\ldots \ldots$. & 5 & 9 & 1 & 0 \\
\hline
\end{tabular}

This patient was one that developed characteristic catatonic symptoms, including flexibilitas cerea and attitudinizing in the last two years of her life. She also had auditory hallucinosis in those years, and showed, as the table indicates, a number of parenchymal losses in the proper area, especially in the superior temporal gyrus. Both the parietal and the temporal lesions were largely infracortical in distribution, in accord with the argument mentioned in the foregoing as to the infracortical origin of catatonia and hallucinosis. It will be noticed from the table that there were comparatively few lesions in the frontal region. The case was one of delusions of jealousy and might have been expected to have shown frontal lesions, which, however, were not greatly in evidence. However, on closer analysis of the delusions, it will be found that they are of a synthetic character and not of a schizophrenic character. They are quasi-normal, and it is not easy to conceive just what histologic basis such delusions ought to have. At all events none of any consequence were found, and the frontal region remains practically destitute of any parenchymal loss and has exceedingly slight evidence of gliosis. Accordingly we may suppose that we are getting histologic evidence in this case merely of the terminal hallucinosis and catatonia and not of the essential paranoia of the patient. 
If we turn to Case 2 (12.41) we find a totally different situation. The amount of parenchymal loss, as indicated by the number of loci, is far larger than in Case 1 (10.9). In fact, there are four times as many foci affected, and the lesions are scattered throughout all three regions - frontal, parietal and temporal. There are relatively more parenchymal losses in the frontal region than in the temporal region and relatively more in the temporal region than in the parietal region.

If we took these data on their face value, we should say that this case ought to be predominantly delusional, but also hallucinatory and catatonic.

There was some question whether the patient was not slightly feebleminded to start with. However, her command of theological verbiage was large, and she was always self-supporting up to the time of her psychosis. My point in mentioning this query is that very possibly some of the apparent parenchymal losses are actually due to an initial lack of cells. The interpretation of an initial lack of cells in any of these areas would of course look in a different direction from that of an initial loss of cells. An initial lack in frontal cells might not at all suggest delusional tendencies; an initial lack of superior temporal cells likewise might have nothing to do with auditory hallucinosis. And a simply constructed parietal mechanism would not necessarily entail disorders of kinesthesia with the production of catatonia.

But taking the parenchymal deficiency as signifying initial loss, let us inquire what the symptoms of this case are. Was it predominantly delusional? She showed hallucinations of a doubtful but probably of a hypnagogic visual nature. It is not clear that she ever had auditory hallucinations. The appearance of parenchymal loss has proceeded in all these areas to a marked degree, so marked, in fact, that it is rather surprising that there was no gross evidence of brain atrophy (the brain weighed $1,130 \mathrm{gm}$., possibly a slight reduction from the normal weight; the patient was cardiac and there was such a condition of infiltration of the brain tissue that we can post hoc readily believe the brain was slightly and diffusely swollen). In short, the lesions in this brain are so numerous and widespread, and so affect both the supracortical and the infracortical regions that correlations of the nature here attempted are impossible. One can find a correlation between almost any symptoms and some properly situated lesion in this case. The patient was deluded, hallucinated and catatonic, and had frontal, temporal and parietal lesions.

Let us, for the sake of argument, regard the frontal lobe delusion correlation as established; let us regard the parieto-occipital correlation with "feelings of being hypnotized" and the "black cat jumping 
over a pitcher" as indicators of disease in the parieto-occipital or occipital areas. How then shall we interpret the absence of auditory hallucinations? Without invoking the poverty of clinical observation and the fact that the patient may have kept auditory hallucinations to herself (she was, in point of fact, often engaged in vituperative language, some of which may have been of hallucinatory origin or of a responsive nature), we must be interested in the fact that some of the temporal lesions shown are exceedingly heavy and affect both the supracortex and the infracortex. Suppose infracortical, temporal lobe lesions of a nature suitable to produce hallucinations (we are still ignorant just what sort of lesion ought to produce hallucinations), would it be too speculative to suggest that the existence of severe supracortical lesions might well interfere with the proper combination and abstraction of the infracortical data in such wise as to produce conscious hallucinations? That is to say, the total supracortical and infracortical mechanism may be conceived to be so altered that, though the conditions for hallucinosis are present in the infracortical zone, the hallucinations are not purveyed through lack of finer connections with the higher psychic apparatus mediated by the supracortical layers. Naturally, I do not wish to claim more than speculative value for such considerations. It is along these lines, however, that $I$ feel that much important correlation will be secured in future.

If we inspect the table of local distribution of lesions in Case 3 (12.47), we find conditions approximating those of Case 2 (12.41), although the duration of Case 3 is far less than that of Case 2. We find here that the parieto-occipital region has numerous lesions and slightly in excess of lesions, also numerous, in the frontal and temporal areas. The case was a catatonic one and in fact, showed both flexibilitas cerea and catatonic stupor, and would probably be classified as a case of catatonic dementia praecox. It appeared that she had auditory hallucinations which she tried to keep out by covering her head with her arms; but the content of these hallucinations was never made out. An attitude of apprehensiveness, an early phase in which she constantly repeated the words "Father Patterson," "Father Patterson," led us to the idea that she had delusions of persecution, or else that she was extremely apprehensive and threw a delusional coloring over every-day features of the environment. The most pronounced regions of cell loss were in the infracortical portions of the parietal and temporal regions.

When we come to the analysis of Case 4 (11.36), we find that the parieto-occipital lesions are far fewer in number, and that the case is more one of losses in the frontal and temporosphenoidal areas. This case, therefore, should show delusions and possibly auditory hallucinations. She never showed convincing catatonic symptoms. 
although she showed a marked mania and hyperkinesis somewhat suggestive of manic-depressive psychosis. She showed characteristic auditory hallucinosis, and it appears that most of her antisocial acts of a mischievous and silly nature were based on these hallucinations. That she showed definite delusions, for example, of persecution or of poisoning, is doubtful. We know little of her history immediately after the onset of mental symptoms which followed confinement in a hospital for consumptives. Throughout her period of observation in a hospital for the insane, she showed elation, erotism, mischievousness and silliness somewhat suggestive of manic-depressive psychosis and again somewhat suggestive of the elation of certain paretics. The cell losses in frontal region were numerous and both supracortical and infracortical in distribution. It is interesting that of all four cases, this one was the case which showed a marked myelin sheath disorder, a disorder so marked as somewhat to suggest the disappearance of fibers in paresis. The supraradiary fibers were absent in all the frontal regions examined and there was a thinning of fibers in the white matter underneath. In short, the disorder in this case was much more than a fine disorder of minor cell mechanisms and approached the more general and globar disorder of paresis, though of course without exudation. On some such lines I would be tempted to try to explain the absence of definite delusions except those based on hallucinations and irritability. I should be tempted to regard the frontal lobe lesions as of such degree that we might think of the case as one that had lost its frontal inhibitions. At the same time, it must be remembered that no coarse atrophy of the brain had supervened, unless we regard the brain weight $-1,140 \mathrm{gm}$. - as demonstrating brain atrophy.

\section{SUMMARY}

Thanks to the work of Elliot Smith, Bolton, Campbell, Brodmann, Ramon y Cajal and others, the neuropathologist can now afford to attempt finer functional histologic correlations in the field of mental diseases, thus aiding in the problems of microlocalization. The antilocalizing tendencies of the Wundtians and the interest in merely logical categories taken by Freudians should not interfere with progress in microlocalization. (Dementia praecox, for example, can be called a matter of maladaptation of the patient to his environment or of the patient to himself and also a disease characterized by cortical changes.

Previous work had shown anomalies in a high proportion of dementia praecox brains and in a correspondingly low proportion of the brains of manic-depressive subjects. (These anomalies may well be interpreted as weak places in these dementia praecox brains, and the 
brains in fact are apt to show scleroses and atrophic processes over and"above the anomalies. But certain perfectly normal-looking brains in dementia praecox also show the same microscopic changes in lesser degrees than are found in the anomalous sclerotic and atrophic brains. The problem of the present communication has been to work out the focality of these microscopic lesions in a few normal-looking brains studied with unusual intensiveness. In the same series of brains, work of previous seasons had shown a dissociation of parenchymatous (neuronic) and interstitial (neuroglia) changes, indicating a tendency on the part of cortex pathology to resemble the pathology of the kidney. But the majority of brains show mixtures of the parenchymatous (neuronic) and the interstitial (neuroglia) lesions. Recent work in comparative anatomy indicates the rather fundamental importance of distinguishing the functions of the upper cortical layers (what may be called the supracortex) from the functions of the lower cortical layers (what may be called the infracortex). The finer processes of mental dissociation (schizophrenia) ought to be correlated with lesions of the supracortex, and such lesions were found in cases with evidence of schizophrenia.

On the other hand, in a case of delusions characterized by no splitting (schizophrenia) whatever, but rather by a process of overelaborate synthesis, there was no evidence of supracortical disorder and in fact, no proposal can be made for any histologic correlate with this process of oversynthesis.

Other processes equally characteristic of dementia praecox, but logically far simpler in their make-up, such as auditory hallucinosis and muscular hypertension (catatonia), received suggestive correlation with processes in the lower layers of the temporal and parietal regions, respectively.

As far as the tissues of these four cases go, there is little or nothing inconsistent in the findings with the hypothesis that ordinary (nonphantastic) delusions are correlated with frontal rather than with otherwise situated lesions; but the supracortical type of delusions found in certain long-standing paranoiacs whose fine mental processes run in a quasi-normal manner, find no special correlation in any region, and the probable lines on which this problem is to be solved remain obscure.

As for auditory hallucinosis, the work seems to afford the expected correlation with temporal lesions. In one case, however, temporal lesions of considerable severity were not attended in life by hallucinations of hearing, but in this case there was also a severe supracortical - disease of the temporal region, and it may be that for the production of hallucinations, some congress is necessary between the activation of the supracortex and infracortex, respectively. 
In previous work on this series, the brains had indicated a postcentral and superior parietal correlation for catatonia whose muscular hypertension was accordingly regarded as very possibly a kind of morbid kinesthesia. Present. work suggests that the anatomic correlate is not merely to the postcentral and parietal regions, but still more specifically to the infracortical parts of these regions.

It may be suggested that the lesions found in samples of tissue in the postcentral, superior parietal, inferior parietal and superior temporal regions indicate a certain systemic tendency in the underlying processes. For these lesions were bilateral and occurred, as it were, in two continuous sheets of tissue on both flanks of the brain in one of the best defined of our cases; these flank lesions were not attended by any similar lesions of the frontal, precentral, occipital and lower temporal and smell regions. The nature of a process which could mildly affect nerve cells and neuroglia on two sides of the brain and also specially affect the infracortical rather than the supracortical portions of these affected sheets of tissue remains a mystery. It is perhaps no greater mystery than that which attends the distribution of lesions in the spinal cord of pernicious anemia. It remains unsettled whether these lesions are secondary in point of time to a noncell-destructive phase in the disease, or whether the lesions of which these microscopic effects are indicators began pari passu with the symptoms - that is, it remains a question whether we are dealing with the excess wear-and-tear process of cell mechanisms morbidly employed or whether the morbidity of neural function is an exact equivalent of the neuronic and neuroglia morbidity. 